\title{
A Facile Approach to Achieve Tunable Wettablility of Graphene-based Films from Superhydrophilicty to Superhydrophobicity
}

Liqiu Zheng ${ }^{1^{*}}$ and Yonglin Zhao ${ }^{2}$

${ }^{1}$ Department of Chemistry and Forensic Sciences, Albany State University, Albany, USA

${ }^{2}$ School of Science, Nantong University, Nantong, China

*Corresponding author: Liqiu Zheng, Department of Chemistry and Forensic Sciences, Albany State University, Albany, USA, Tel: 0012294207052; E-mail: Liqiu.Zheng@asurams.edu

Received date: January 6, 2018; Accepted date: February 2, 2018; Published date: February 9, 2018

Copyright: (c) 2018 Zheng L, et al. This is an open-access article distributed under the terms of the Creative Commons Attribution License, which permits unrestricted use, distribution, and reproduction in any medium, provided the original author and source are credited.

\begin{abstract}
A facile, safe and low cost thermal approach was developed to regulate wettability of graphene-based surfaces from superhydrophilicity to superhydrophobicity, which promises mass production on surface technology in various industrial applications. The long-lasting stable superhydrophobicity was attained by hydrogenating graphene with chem-absorbed functional groups. Meanwhile, the approach paves the way for producing fully hydrogenated graphene, which is an entirely novel material called graphene.
\end{abstract}

Keywords: Wettability; Superhydrophilicity; Superhydrophobocity; Hydrogenation; Graphane

\section{Introduction}

Graphene, one of the thinnest materials known [1], is a single-atomthick sheet comprised of sp2-hybridized carbon atoms, arranged in a hexagonal lattice. Thanks to its unique single-atom structure, graphene is a complete surface material in which virtually all atoms are exposed to the environment, which provides a fertile playground for researchers to manipulate its surface properties, for instance, tailor its wettability from wetting to non-wetting, in both theoretical and experimental fields. However, extensive research has been mainly focused on photoelectric performance of graphene, because of its other extraordinary features which find numerous novel device applications. For instance, its excellent electronic transport performance is widely utilized in electronics fields [2]; robustness and light weight in micromechanical resonators [3]; high conductivity in electrical batteries [4]; high surface-to-volume ratio, atomic thinness in field emitters [5] and optical transparency in transparent membranes for electron microscopy. In contrast, far less attention has been devoted to the properties of graphene surfaces, although controllable manipulation of wetting behaviors on graphene surface promises equally significant technological applications in various fields such as micro-fluidics, water harvesting, anti-fouling, and anti-corrosion [6]. As far as wettability is concerned, the more striking feature of conformal graphene coatings is wetting transparency, which could exist when mono-layer graphene is deposited on the appropriate substrates (such as copper, gold or silicon) [7-9], which refers to the fact that Van der Waals forces between water and substrate are able to be seen through graphene layer, due to the extreme thinness of graphene. The wetting transparency of graphene enables even wider applications in which electronic and wetting properties can be independently controlled, leading to the design of multifunctional smart surfaces.

Currently, some attempts have been prompted to control wettability of graphene films. Surface properties are governed by surface physical architecture and chemical composition, which leads to two directions: one is to engineer morphological patterns on surfaces; the other is to alter surface chemical composition. Laser interference and lithographic techniques are mostly taken to fabricate patterned roughness on graphene to realize desired wettability. These techniques require costly sophisticated equipment and fussy manipulation [10,11]. Consequently, altering chemical composition through functionalizing surface predominates in tuning wettability of graphene. For example, Rafiee et al. [12] developed a technique to regulate wettability of graphene films from super hydrophilicity to super hydrophobicity by sonicating graphene in water and acetone solutions with different concentration. Although a controllable contact angle (CA) changes from $0^{\circ}$ to $160^{\circ}$, physic-absorbed functional groups by sonication are not stably grafted onto graphene films which results in a quick loss of super hydrophobicity. Meanwhile, long time sonication of graphene films produces lots of undesired defects. Fluorinated graphene $[13,14]$ is another effective but cumbersome approach which produces very limited yield and offers no avenue for scale up. Additionally, in the process $\mathrm{XeF}_{2}$ and $\mathrm{HF}$ are involved which pose serious safety issues. Therefore, it is imperative to develop a facile, inexpensive, safe and scalable approach to achieve tunable wettability of graphene films for practical applications.

In this contribution, for the first time we reported a facile method to tune the wettability of graphene-based films from super hydrophilicity to super hydrophobicity. This low cost, safe approach is of vital technological importance because of its scalability which would promise various industrial applications, ice mitigation, advanced multifunctional coatings and composites [15,16]. More importantly, the approach holds great potential for transforming graphene into an entirely novel material called graphane when fully hydrogenated, which would find even wider applications in different fields.

\section{Materials and Methods}

A few layers of graphene powder (Angstrom Materials LLC, $1.5 \mathrm{~g}$, carbon content 97\%) was sonicated in $120 \mathrm{~mL}$ of a $3: 1$ mixture of $\mathrm{H}_{2} \mathrm{SO}_{4}(18 \mathrm{M})$ and $\mathrm{HNO}_{3}(17 \mathrm{M})$ at $40^{\circ} \mathrm{C}$ for 4 hours. The dispersion 
was allowed to stand at room temperature for 3 days. After repeatedly washed with water (total $4 \mathrm{~L}$ ), by centrifugation and decantation, the oxidized graphene was filtered through a $0.2 \mu$ Polytetrafluoroethylene (PTFE) membrane, with a final ethanol wash. The product was allowed to dry under vacuum overnight. The dried product was dissolved in 50 $\mathrm{mL}$ of Dimethylformamide (DMF) (Fisher Chemical $\geq 99.8 \%$ ). The total amount of as-produced graphene oxide was divided into 4 portions with equal amount for 4 different batches of samples. All portions were air-brushed onto quartz slides and then placed on a heating plate at $150^{\circ} \mathrm{C}$ in order to remove the DMF solvent from coating. Instantly, the first batch, graphene oxide, was obtained. The other 3 batches was ready for further treatment. Two batches of quartz slides with graphene coating were put into a tubing furnace under hydrogen $\left(\mathrm{H}_{2}\right)$ flow with $20 \mathrm{ml} / \mathrm{min}$. After 30 mins of reduction at $350^{\circ} \mathrm{C}$, one batch was taken out for various characterizations. Then after another 30 mins, the second batch was taken out. Next, a preprepared $\mathrm{Ni}(8 \% \mathrm{wt}) / \mathrm{Al}_{2} \mathrm{O}_{3}$ catalyst in a graphite boat was placed upstream of the $\mathrm{H}_{2}$ flow (Note: Preparation for catalyst: The nickel nitrate hydrate $\left(\mathrm{Ni}\left(\mathrm{NO}_{3}\right)_{2} \cdot 6 \mathrm{H}_{2} \mathrm{O}\right)$ solution with $\mathrm{Al}_{2} \mathrm{O}_{3}$ powder (BET $270 \mathrm{~m}^{2} / \mathrm{g}$ ) was ultra-sonicated and dried at $110^{\circ} \mathrm{C}$, ground to a fine powder, and then calcined at $500^{\circ} \mathrm{C}$ for 2 hours). The catalystcontaining graphite boat and the last batch of graphene coating quartz slides were kept 5 inches apart. The temperature was raised to $800^{\circ} \mathrm{C}$ and maintained for 200 mins. The final step was to naturally cool down the furnace to room temperature under $\mathrm{H} 2$ flow. Wettability was evaluated by water contact angle goniometry. Morphological structure information was revealed by scanning electron microscopy (SEM) (JEOL JSM-7000F), transmission electron microscopy (TEM) (JEOL JEM-2100F) and atomic force microscopy (AFM) (Bruker Nano). Chemical composition was scrutinized by Fourier transform infrared spectroscopy (FTIR) (Agilent Cary 630) and Raman Spectroscopy (Horiba Jobin Yvon Lab Ram 800).

\section{Results and Discussion}

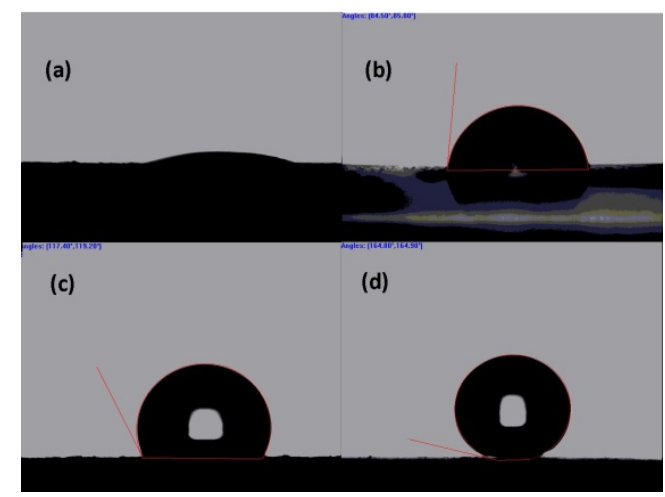

Figure 1: Wettability properties of differently treated graphenebased films. (a) As-produced graphene-based film; (b) graphenebased film with 30-min reduction; (c) graphene-based film with 60min reduction; (d) Hydrogenated graphene-based film.

Wettability of samples was characterized by CA measurements. CA is a conventionally measured angle between a tangential line along a liquid-vapor interface and a base line where the liquid meets a solid surface. It quantifies wettability of a solid surface. If CA $>90^{\circ}$ the solid surfaces are categorized as hydrophobicity. If $\mathrm{CA}<90^{\circ}$, the surfaces are hydrophilicity. For the extreme cases, if $\mathrm{CA}>150^{\circ}$ surfaces are called a super hydrophobicity and if $\mathrm{CA}<10^{\circ}$, surfaces are a super hydrophilicity [17]. Vastly different wettability was demonstrated by graphene-based coatings in Figure 1. Figure 1a displays the super hydrophilicity of as-produced graphene-based surface because CA is much smaller than $10^{\circ}$. A water droplet deposited onto the surface instantly spread out and formed a water film, revealing an abundantly hydrophilic nature of as-produced graphene-based (graphene oxide) plane. Figure $1 \mathrm{~b}$ depicts slightly reduced graphene-based surfaces with the CA of $84^{\circ} \pm 2^{\circ}$ after 30 mins reduction, an obvious increase in CA observed. Although the surface is still in the hydrophilic range, the hydrophilic nature was greatly weakened by 30 mins reduction treatment. After further treatment by extending the reduction time to 60 mins, the water CA increased to $117^{\circ} \pm 2^{\circ}$ as seen in Figure 1c which falls into the hydrophobic category. The results are consistent with previous reports [18]. The inherent hydrophobic nature of graphene sheet dominated after the thermal removal of hydrophilic functional groups, which altered the surface to be hydrophobic. But there was no noticeable increase in CA observed on those graphene films when the reducing time extends even longer than 200 mins (for instance, the graphene films were reduced at $800^{\circ} \mathrm{C}$ without catalyst. Not shown here). In contrast, hydrogenation caused a dramatic change in wettability on the surface because CA increased to $164^{\circ} \pm 2^{\circ}$ as revealed in Figure 1d, which is much larger than $150^{\circ}$. Additionally, the contact angle of $164^{\circ} \pm 2^{\circ}$ was measured after the samples were exposed to ambient conditions for 2 months. Super hydrophobicity was achieved after hydrogenation. $\mathrm{Ni}(8 \% \mathrm{wt}) / \mathrm{Al}_{2} \mathrm{O}_{3}$ catalyst breaks down the hydrogen-hydrogen molecule bond into atomic hydrogen. These atomic radicals combine with dangling carbon bonds which are left behind by the elimination of other groups forming $\mathrm{C}-\mathrm{H}$ bonds. The chemically absorbed functional groups $-\mathrm{CH}_{2}$ or $-\mathrm{CH}_{3}$ onto graphene sheet induce a long lasting super hydrophobicity. Tunable wettability from super hydrophilicity to super hydrophobicity was achieved by this facile, low cost, and safe approach which is ready for mass production.

As aforementioned, surface morphological roughness and chemical composition play parts in surface properties. In order to examine surface structure, samples were thoroughly scrutinized by SEM, AFM and TEM. As an ideal two dimensional material, graphene has even higher specific surface area than its allotrope carbon nanotubes [19]. Its lateral sheet can extend up to tens of micrometers as revealed by all images in Figure 2. Figure 2a discloses that micron-sized graphene oxide sheets have a flaky texture, reflecting their layered microstructure. Air-brushed graphene films created inherent roughness which stems from random arrangement of graphene sheets. Figures $2 \mathrm{~b}$ and $2 \mathrm{c}$ portray morphology of graphene-based sheets after reduction 30 minutes and 60 minutes, respectively. Figure $2 \mathrm{~d}$ depicts the surface structure of the hydrogenated graphene. All SEM images suggest that the high aspect ratio and large nominal surface areas of flaky graphene were not altered by reduction/hydrogenation treatments. All the graphene-based films demonstrate a structure of individual flakes with highly wrinkled, folded and buckled edges, which generated different scale roughness that can be down to nanosize. The average surface roughness established hierarchical structures on the films. 
Citation: Zheng L, Zhao Y (2018) A Facile Approach to Achieve Tunable Wettablility of Graphene-based Films from Superhydrophilicty to Superhydrophobicity. J Nanosci Curr Res 6: 118. doi:10.4172/2572-0813.1000118

Page 3 of 6

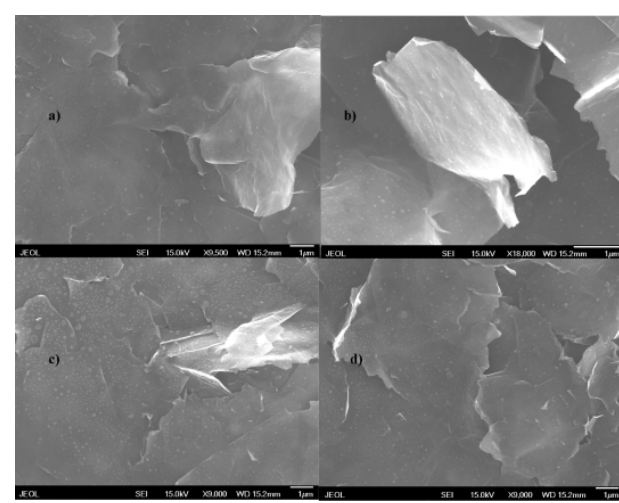

Figure 2: Scanning electron microscopy (SEM) images (a) asproduced graphene-based film; (b) graphene-based film with 30min reduction (b) graphene-based film with 60 -min reduction; c) hydrogenated graphene-based film.

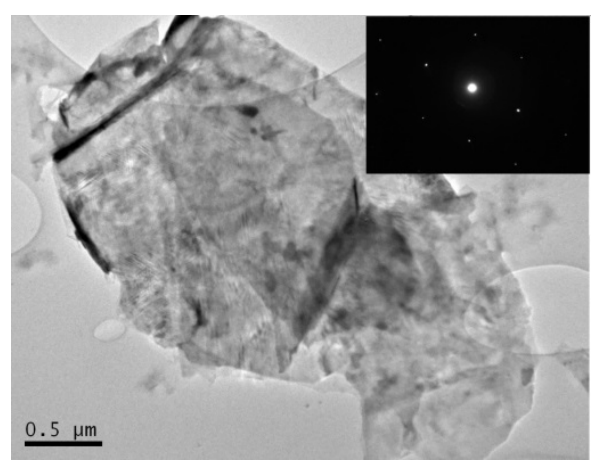

Figure 3: Transmission electron microscopy (TEM) image for hydrogenated graphene-based film; Insert is reciprocal lattice peaks obtained by $2 \mathrm{D}$ Fourier transform based on TEM image for hydrogenated graphene-based film.

TEM in Figure 3 further confirms that the flaky transparent feature of graphene-based film was maintained even after hydrogenation. Particularly, it is worth mentioning that hydrogenation did not change the hexagonal arrangement of carbon atoms as characterized in the insert of Figure 3, in which reciprocal lattice peaks were obtained by 2D Fourier transform based on TEM image of the hydrogenated graphene-based film.
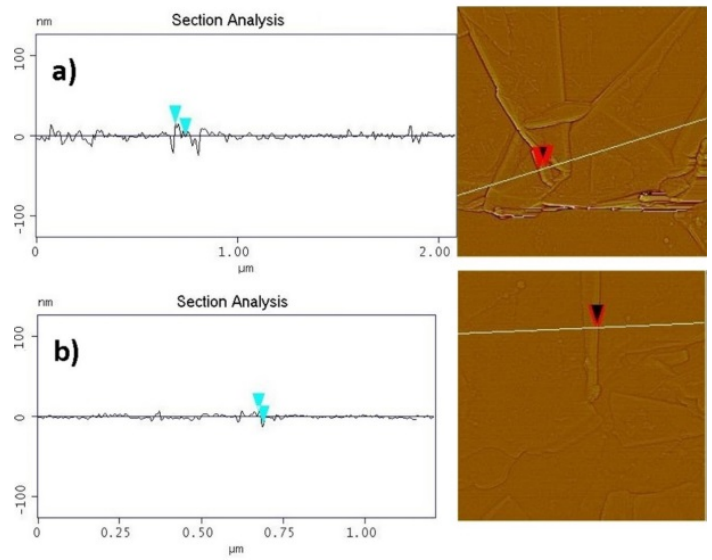

Figure 4: Atomic force microscopy(AFM) images (a) graphenebased film with 30-min reduction (b) hydrogenated graphene-based film.

AFM images further corroborate the morphological analysis. As revealed by SEM images, AFM images in Figure 4 substantiate similar graphene flakes with randomly stacked and crumpled surface structure. The roughness on surfaces is up to ten nanometers on individual graphene sheet, consistent with SEM results. Figure $4 \mathrm{a}$ demonstrates morphology of graphene-based film after 30 minutes reduction and Figure $4 \mathrm{~b}$ presents the surface structure of graphenebased film after hydrogenation. The comparison of both AFM images shows that reduction and hydrogenation procedures did not result in any noticeable changes in the structural morphology. It is important to note that increased time reduction does not make any difference in morphology when comparing SEM of graphene-based film after 60 minutes reduction in Figure 2b with AFM of graphene-based film after 30 minutes reduction in Figure $4 \mathrm{a}$.

In spite of similar surface morphology, samples display strikingly different wetting behaviors. To clarify the underlying reason behind this phenomenon, FTIR was adopted to probe surface chemical composition. Figure 5a depicts the transmittance spectra of graphene oxide films in which characteristic peaks of graphene oxide correspond to: alkoxy C-O $\left(1116 \mathrm{~cm}^{-1}\right)$; epoxy C-O $\left(1228 \mathrm{~cm}^{-1}\right)$; stretching single bond carboxy $\mathrm{C}-\mathrm{O}\left(1414 \mathrm{~cm}^{-1}\right)$; stretching double bond carboxy $\mathrm{C}=\mathrm{O}$ $\left(1735 \mathrm{~cm}^{-1}\right)$. While a broad peak is supposed to appear at $3442 \mathrm{~cm}^{-1}$ which is ascribed to the water $\mathrm{O}-\mathrm{H}$ stretching vibration. Notably, this peak blue-shifted above $3500 \mathrm{~cm}^{-1}$ in graphene oxide as seen in Figure $5 \mathrm{a}$ on the grounds that the stretching bond became very rigid after the loss of electrons to strong acid which is in good agreement with other reported results in the literatures [20]. The presence of oxygen functionalities on the basal plane carbon atoms rendered the asproduced graphene-based film a super hydrophilic nature, which explains why water droplets spread into a thin film instantly when deposited on graphene oxide films. It is worth pointing out that the graphene oxide undergoes acid treatment which considerably increased wettability of samples since it brought additional polar $\mathrm{OH}$ groups and oxygen functional groups like carboxyl and epoxyl onto the surface of grapheme [21]. On the other hand, acid treatment created defects in graphene which opens possibilities for functional groups addition and thus varying properties around defect sites. 


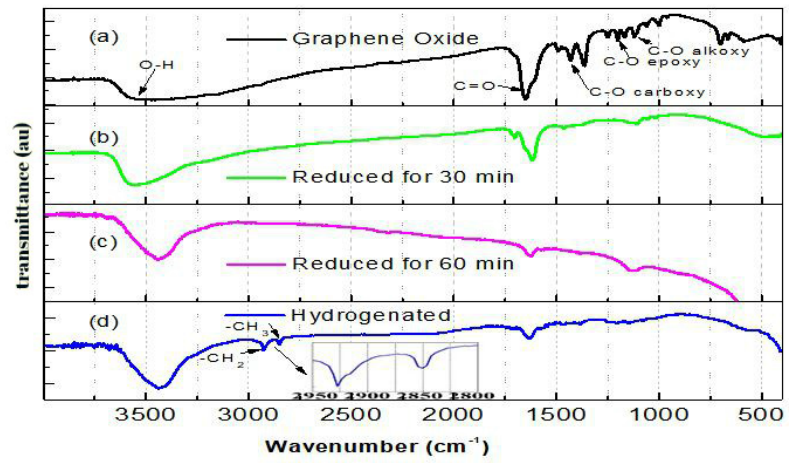

Figure 5: FT-IR transmittance spectra (a) as-produced graphenebased film (b) graphene-based film with 30-min reduction(c) graphene-based film with 60-min reduction (d) hydrogenated graphene-based film and hydrogenated peaks were revealed by the insert in (d).

As observed in Figure 5b, after 30 min reduction, some oxygen functional groups like carboxy $\mathrm{C}=\mathrm{O}\left(1735 \mathrm{~cm}^{-1}\right)$ dramatically decreased while others like carboxy C-O $\left(1414 \mathrm{~cm}^{-1}\right)$ were completely eliminated which accounts for the increase in CA from less than $10^{\circ}$ to $84^{\circ} \pm 2^{\circ}$. It suggests that the hydrophilic functional groups on the surface of graphene films were partially removed. In addition, 30-min reduction did not soften the broad peak of $\mathrm{O}-\mathrm{H}$ stretching mode and no red shift was observed because it is still above $3500 \mathrm{~cm}^{-1}$ as indicated in Figure 5b. Further reduction of 60 mins resulted in the riddance of virtually all oxygen functional groups as shown by Figure 5c. Accordingly, CA increased to $117^{\circ} \pm 2^{\circ}$. The elimination of carboxyl and hydroxyl groups from basal plane of graphene allowed an intrinsically hydrophobic nature of graphene unearthed which was also reflected by a strong tendency of graphene powder to agglomerate in aqueous solvents after 60 mins reduction. Although there was a recognizable difference in CA between 30 mins and 60 mins reduction treatments, CA remained $117^{\circ} \pm 2^{\circ}$ even when reduction time extended longer than 200 minutes. Therefore $164^{\circ} \pm 2^{\circ} \mathrm{CA}$ on hydrogenated graphene-based film did not result from the longer treatment time (200 mins) of hydrogenation but the presence of catalyst $\mathrm{Ni}(8 \% \mathrm{wt}) / \mathrm{Al}_{2} \mathrm{O}_{3}$, which made atomic hydrogen available by breaking down hydrogen-hydrogen molecule bonds and accomplishes the decoration of functional groups $-\mathrm{CH}_{2}$ or $-\mathrm{CH}_{3}$ onto graphene sheet as displayed Figure 5d. Particularly, the insert in Figure 5d clearly discloses that additional peaks $2926 \mathrm{~cm}^{-1}$ and $2853 \mathrm{~cm}^{-1}$ are the stretching modes of $\mathrm{H}$ bonded to olefinic carbon and the bending modes of $\mathrm{H}$ bonded to aromatic carbon, respectively. The introduction of hydrogenated groups lowers surface energy of graphene sheet, leading to a stable super hydrophobicity, which explains why CA reached $164^{\circ} \pm 2^{\circ}$ after hydrogenation due to the fact that grafted hydrocarbon groups enhance hydrophobicity of the graphene surface. Remarkably, the broad water peak of $\mathrm{O}-\mathrm{H}$ stretching mode, after gaining electrons, softened down to $3442 \mathrm{~cm}^{-1}$ as shown in Figures $5 \mathrm{c}$ and $5 \mathrm{~d}$. The adsorption of oxygen functionalities or hydrogen groups on graphene can be used to tailor wettability properties of graphenebased films, as already suggested for the modified wetting graphene films which are sonicated in acetone [12]. Tunable wettability from super hydrophilicity to super hydrophobicity was achieved with a facile and inexpensive approach which holds promise for mass production and creating novel graphane.

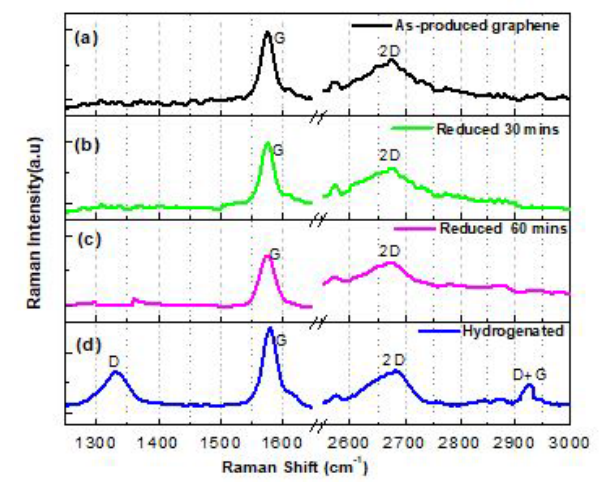

Figure 6: Raman spectra (a) as-produced graphene-based film (b) graphene-based film with 30-min reduction(c) graphene-based film with 60-min reduction (d) hydrogenated graphene-based film.

Raman spectroscopy decisively substantiates the FTIR results. As revealed in all images in Figure 6, there are two intense characteristic peaks of graphene, which are $\mathrm{G}$ band at $1580 \mathrm{~cm}^{-1}$ and $2 \mathrm{D}$ band at $2700 \mathrm{~cm}^{-1}$. The $\mathrm{G}$ band is due to the in-plane vibrational (E2g) mode, while the $2 \mathrm{D}$ band results from the two phonon inter-valley double resonance scattering [22,23]. It is worth pointing out that the red-shift of the $2 \mathrm{D}$ band to below $2700 \mathrm{~cm}^{-1}$ at around $2685 \mathrm{~cm}^{-1}$ is caused by multilayer of graphene for Raman characterization. The $2 \mathrm{D}$ peak of one-layer graphene would show up at $2700 \mathrm{~cm}^{-1}$. The number of layer of graphene causes red-shift because the $2 \mathrm{D}$ band acts as the fingerprint in distinguishing one-layer or multilayer. Compare with Figures $6 \mathrm{a}-6 \mathrm{c}$, besides $\mathrm{G}$ and $2 \mathrm{D}$ bands, there are two additional peaks which are assigned to $D\left(1340 \mathrm{~cm}^{-1}\right)$ and $D+G\left(2920 \mathrm{~cm}^{-1}\right)$ bands as shown in Figure $6 \mathrm{~d}$. The observation of $\mathrm{D}$ and $\mathrm{D}+\mathrm{G}$ peaks amply illuminates that defects were introduced onto the graphene lattice by the hydrogenation treatment, which were the newly formed $\mathrm{C}-\mathrm{H}$ sp3 bonds and the breaking of the translational symmetry of $\mathrm{C}=\mathrm{C} \mathrm{sp} 2$ bonds [24]. CA for a smooth surface can be expressed in the form of Young's equation $[25,26]$ :

$\cos \theta=\left(\gamma_{s v}-\gamma_{s l}\right) / \gamma$

In equation (1), surface energies of solid-liquid, solid-vapor and liquid-vapor interfaces are denoted by $\gamma s v, \gamma s l, \gamma$, respectively. To describe textured surfaces there are two models known as "Wenzel" [27] in equation (2) and "Cassie-Baxter" in equation (3) [28].

$$
\begin{aligned}
& \cos \theta^{*}=r \cos \theta \\
& \cos \theta^{*}=f_{A} \cos \theta_{A}+f_{B} \cos \theta_{B}
\end{aligned}
$$

In equation (2) Wenzel model, $\theta^{*}$ is the effective CA on the textured surface; $r$ is the roughness ratio; and $\theta$ is the Young CA on a flat solid surface. In equation (3) Cassie-Baxter model, $\theta$ is the effective CA; $f A$ and $f B$ are fractions of surface area of components $A$ and $B ; \theta_{A}$ and $\theta_{B}$ are the Young CAs on flat surfaces of $A$ and $B$, respectively. The difference between two models is whether the liquid drop retains intimate contact with a solid surface at all points or whether the liquid bridges across surface protrusions, thus, resulting in a drop sitting on a 
Page 5 of 6

composite solid and vapor surface, suggesting that the textured surface must have the capability of entrapping air pockets. The former is a Wenzel model which applies to hydrophilicity or mild hydrophobicity. The latter is a Cassie-Baxter model which applies to strong or super hydrophobicity. Due to high surface energy of surfaces, the strong adhesion between graphene surfaces and water droplet compels an intimate contact which does not allow air being entrapped between those textures. Hence, equation (2) Wenzel model explains CAs from smaller than $10^{\circ}$ in Figure 1a to $117^{\circ} \pm 2^{\circ}$ in Figure 1c. The actual CA is mainly determined by the Young CA on the flat surface and amplified by the roughness ratio $r$. It suggests that graphene oxide surfaces with abundant hydrophilic groups, along with 30 mins reduced graphenebased surfaces, still remains hydrophilic even with certain roughness. After 60 mins reduction, an intrinsic hydrophobicity is brought to light by the removal of nearly all hydrophilic groups. The CA remains the same after the complete exposure of intrinsic hydrophobicity of graphene-based film by even longer-time reduction. CA can be further enhanced by lowering surface energy when altering surface chemical composition [29]. Reportedly, the introduction of functional groups like - $\mathrm{CF} 3,-\mathrm{CH} 3$ and $-\mathrm{CH} 2$ could effectively lower surface energy, realizing super hydrophobicity [30]. For super hydrophobicity, CassieBaxter equation (3) is adopted to describe strong or super hydrophobicity, in which there are three requirements optimizing surface conditions and thus maximizing CA. The first one is that the surface must have an ability to capture air pockets in its architectures. When the surface is capable of entrapping air bubbles, fractions of surface area of components $\mathrm{A}$ and $\mathrm{B}$ will be a solid portion and an air portion. The $\mathrm{CA} B$ in air is $180 \mathrm{o}$ (then $\cos \theta_{B}=-1$ ) and $f_{B}=1-f_{A}$ which leads to the simplification of equation (3) as: $\cos \theta^{*}=f_{A} \cos \theta_{A}-\left(1-f_{A}\right)(4)$

Then after further simplifying, equation (4) becomes more compact as:

$$
\cos \theta^{*}=f_{A}\left(\cos \theta_{A}+1\right)-1(5)
$$

In order to obtain a maximal CA, the larger portion of water sitting on the air bubble leads to the higher effective CA suggesting the second optimal condition is that a smaller $f_{\mathrm{A}}$ is required. The third one is that the Young $\mathrm{CA}_{\mathrm{A}}$ on the solid portion is as large as possible which could maximize the effective CA. In the hydrogenated graphene-based film's case, the topology of graphene flakes, together with weak adhesion induced by low surface energy, allows air to be entrapped within wrinkles or folds underneath a water drop which satisfies the first optimal condition. The second condition for maximal $\mathrm{CA}$ is that the fraction of solid portion $f_{\mathrm{A}}$ should be minimized, which implies the larger portion of a water droplet would sit on air which allows for $180^{\circ}$. As one of the thinnest known materials, the folded/ wrinkled/buckled edges of graphene flakes protrude on surfaces supporting water droplets which drastically decrease the contact area of a droplet with the nano-protrusions. In other words, a smaller area fraction, fA, is realized by the morphologically thin character of graphene, which meets the second optimal requirement. The third optimal condition requires a larger ${ }_{A}$, the Young CA on a flat surface, which can be fulfilled merely by lowering the surface energy. Acid treatment opens possibilities for functional groups addition which enhances the reactivity for functionalizing them with low surface energy groups. As a result, with catalyst breaking $\mathrm{H} 2$ molecule bond into atomic hydrogen, functional groups $-\mathrm{CH} 2$ or $-\mathrm{CH} 3$ are formed and then chemically absorbed onto the graphene sheet, leading to a long lasting stable super hydrophobicity. It implies that the decoration of $-\mathrm{CH}_{2}$ or $-\mathrm{CH}_{3}$ groups onto graphene films lowers the surface energy which yields a larger $C A$ and thus achieves the third optimizing condition.

Therefore, apart from the geometrical microstructure, the chemical composition of the surface also plays an important role in wettability properties of graphene-based films. The random stacking of graphene sheets introduces inherent roughness, along with modulating surface chemical composition, which suffices to regulate wettability from super hydrophilicity to super hydrophobicity.

\section{Conclusion}

In summary, the thermal reduction of graphene-based films is accompanied by the elimination of epoxy, carbonyl/carboxyl groups and the introduction of hydrocarbon groups, which can regulate wettability of graphene-based surfaces from super hydrophilicity to super hydrophobicity. By varying the reduction time, the amount of those hydrophilic groups plays a part in tailoring wettability of the surfaces. A facile and low cost approach to hydrogenate graphene with $\mathrm{Ni}(8 \% \mathrm{wt}) / \mathrm{Al} 2 \mathrm{O} 3$ catalyst, readily realizes super hydrophobicity which meantime promises for scaled-up production. If all of the oxygen groups are removed, and the sample becomes fully hydrogenated, an even higher CA can be achieved. More importantly, a completely novel material named graphane is potentially created by fully hydrogenating graphene. Thanks to its low mass and large surface area, along with other exceptional properties, graphene-based film with tunable wettability could be used as a multifunctional coating material for various applications in solar panel, aerospace, aviation, and even antiicing fields.

\section{References}

1. Si Y, Samulski ET (2008) Synthesis of water soluble graphene. Nano Lett 8: 1679-1682.

2. Jana S, Singh JK, Kwak SK (2009) Vapor-liquid critical and interfacial properties of square-well fluids in slit pores. J Chem Phys 130: 214707.

3. Chen C, Rosenblatt S, Bolotin KI, Kalb W, Kim P, et al. (2009) Performance of monolayer graphene nanomechanical resonators with electrical readout. Nat Nanotechnol 4: 861-868.

4. Casolo S, Løvvik OM, Martinazzo R, Tantardini GF (2009) Understanding adsorption of hydrogen atoms on grapheme. J Chem Phys 130: 054704-054714.

5. Eda G, Unalan HE, Rupesinghe N, Amaratunga AGG, Chhowalla M (2008) Field emission from graphene based composite thin films. Appl Phys Lett 93: 233502-233505.

6. $\mathrm{Hu} \mathrm{HW}$, Allen $\mathrm{CK}$, Li JH, Kong YY, Wang XW, et al. (2014) Multifunctional organically modified graphene with superhydrophobicity. Nano Res 7: 418-433.

7. Kim GT, Gim SJ, Cho SM, Koratka N, Oh IK (2014) Wetting-transparent graphene films for hydrophobic water-harvesting surfaces. Adv Mater 26: 5166-5172.

8. Mugele F (2012) Wetting: Unobtrusive graphene coatings. Nat Mater 11: 182-183.

9. Rafiee J, Mi X, Gullapalli H, Thomas AV, Yavari F, et al. (2012) Wetting transparency of grapheme. Nat Mater 11: 217-222.

10. Huang YY, Chen XD, Zhang MQ (2014) Reversible surface wettability conversion of graphene films: optically controlled mechanism. J Mater Sci 49: 3025-3033

11. Naghdi S, Jaleh B, Shahbazi N (2016) Reversible wettability conversion of electrodeposited graphene oxide or titania nanocomposite coating: Investigation of surface structures. Appl Surf Sci 368: 409-416. 
Citation: Zheng L, Zhao Y (2018) A Facile Approach to Achieve Tunable Wettablility of Graphene-based Films from Superhydrophilicty to Superhydrophobicity. J Nanosci Curr Res 6: 118. doi:10.4172/2572-0813.1000118

Page 6 of 6

12. Rafiee J, Rafiee MA, Yu ZZ, Koratkar N (2010) Super hydrophobic to superhydrophilic wetting control in graphene films. Adv Mater 22: 2151-2154.

13. Galande C, Gao W, Mathkar A, Dattelbaum AA, Narayanan TN, et al. (2014) Science and engineering of graphene Oxide. Part Part Syst Charact 31: 619-638.

14. Jayaramulu K, Datta KKR, Rosler C, Petr M, Otyepka M, et al. (2016) Biomimetic superhydrophobic/superoleophilic highly fluorinated graphene oxide and zif- 8 composites for oil-water separation. Angew Chem Int Ed 55: 1178-1182.

15. Feng X, Jiang L (2006) Design and creation of superwetting/antiwetting surfaces. Adv Mater 18: 3063-3078.

16. Wang JN, Zhang YL, Liu Y, Zheng WH, Lee LP, et al. (2015) Recent developments in superhydrophobic graphene and graphene-related materials: from preparation to potential applications. Nanoscale 7: 7101-7114.

17. Drelich J, Chibowski E (2010) Superhydrophilic and superwetting surfaces: Definition and mechanisms of control. Langmuir 26: 1862118623.

18. Hsieh CT, Yang BH, Tzou DY, Chen YF, Chen WY (2013) Liquid repellency from graphite sheets with different oxidation levels. Thin Solid Films 529: 80-84.

19. Wang G, Yang J, Park J, Gou X, Wang B, et al. (2008) Facile synthesis and characterization of graphene nanosheets. J Phys Chem C 112: 8192-8195.
20. Chambers A, Park C, Terry R, Baker K, Rodriguez MN (1998) Hydrogen storage in graphite nanofibers. J Phys Chem B 102: 447-4482.

21. Chen W, Yan L (2010) Preparation of graphene by a low-temperature thermal reduction at atmosphere pressure. Nanoscale 2: 559-563.

22. Kostiuk D, Bodik M, Siffalovic P, Jergel M, Halahovets Y, et al. (2016) Reliable determination of the few-layer graphene oxide thickness using Raman spectroscopy J Raman Spectr 47: 391-394.

23. Luo ZQ, Yu T, Kim KJ, Ni ZH, You YM, et al. (2009) Thickness-dependent reversible hydrogenation of graphene layers. ACS Nano 7: 1781-1788.

24. Elias DC, Nair RR, Mohiuddin TMG, Morozov SV, Blake P, et al. (2009) Control of graphene's properties by reversible hydrogenation: Evidence for graphane. Science 323: 610-613.

25. Adamson AW (1990) Physical chemistry of surfaces (5th edn.). WileyInterscience, New York.

26. Moskovits M (1985) Surface-enhanced spectroscopy. Rev Mod Phys 57: 783.

27. Wenzel RN (1936) Resistance of solid surfaces to wetting by water. Ind Eng Chem 28: 988.

28. Cassie ABD, Baxter S (1944) Wettability of porous surfaces. Trans Faraday Soc 40: 546-561.

29. Quere D (2008) Wetting and Roughness. Annu Rev Mater Res 38: 71-99.

30. Hare EF, Shafrin EG, Zisman WA (1954) Properties of films of adsorbed fluorinated acids. J Phys Chem 58: 236-239. 